\title{
ASYMPTOTIC BEHAVIOUR, NODAL LINES AND SYMMETRY PROPERTIES FOR SOLUTIONS OF SUPERLINEAR ELLIPTIC EQUATIONS NEAR AN EIGENVALUE*
}

\author{
Dimitri Mugnai ${ }^{1}$
}

\begin{abstract}
We give the precise behaviour of some solutions of a nonlinear elliptic B.V.P. in a bounded domain when a parameter approaches an eigenvalue of the principal part. If the nonlinearity has some regularity and the domain is for example convex, we also prove a nonlinear version of Courant's Nodal theorem.
\end{abstract}

Mathematics Subject Classification. 35B40,35J65.

Received March 30, 2004. Revised November 30, 2004.

\section{INTRODUCTION}

In a celebrated paper, Z.Q. Wang proved that, if $\Omega$ is a bounded and smooth domain of $\mathbb{R}^{N}$ and $g: \mathbb{R} \longrightarrow \mathbb{R}$ is a $C^{1}$ superlinear and subcritical function such that $g(0)=g^{\prime}(0)=0$, then the nonlinear Dirichlet problem

$$
\begin{cases}-\Delta u=g(u) & \text { in } \Omega, \\ u=0 & \text { on } \partial \Omega,\end{cases}
$$

has at least three nontrivial solutions (see [23]). In the same spirit, in [19] it was proved that, if $g: \Omega \times \mathbb{R} \longrightarrow \mathbb{R}$ is a superlinear and subcritical Carathéodory's function, then there exists $\delta_{i}>0$ such that $\forall \lambda \in\left(\lambda_{i}-\delta_{i}, \lambda_{i}\right)$, $i \geq 2$, the problem

$$
\begin{cases}-\Delta u-\lambda u=g(x, u) & \text { in } \Omega, \\ u=0 & \text { on } \partial \Omega\end{cases}
$$

has at least three nontrivial solutions (here $\left(\lambda_{i}\right)_{i}$ denotes the sequence of eigenvalues of $-\Delta$ on $H_{0}^{1}(\Omega)$ ). In particular in [19] it was proved that two of such solutions have positive energy, approaching 0 as $\lambda \rightarrow \lambda_{i}^{-}$. In this paper we want to prove that such solutions have some finer properties, inherited by the fact that $\lambda$ is near an eigenvalue and by the fact that their energy approaches 0 . In particular our results are related to the behaviour of the nodal lines of such solutions (see Th. 2.1) and to some symmetry properties when $g$ and $\Omega$ have some symmetries (see Th. 2.2).

\footnotetext{
Keywords and phrases. Eigenvalues, $L^{\infty}-H_{0}^{1}$ estimate, nodal lines, symmetries.

* Research supported by the M.I.U.R. project Metodi Variazionali ed Equazioni Differenziali Nonlineari.

1 Dipartimento di Matematica e Informatica, Università di Perugia, via Vanvitelli 1, 06123 Perugia, Italy;

mugnai@dipmat .unipg.it
}

(c) EDP Sciences, SMAI 2005 
To our knowledge, there are not many results concerning nodal lines of sign-changing solutions, as the ones we are considering. For sure, remarkable results can be found in $[4,6,7,10]$. In $[6]$, the authors assume to deal with a function $g: \Omega \times \mathbb{R} \longrightarrow \mathbb{R}$ of class $C^{1}$ such that

$\overline{\left(g_{1}\right)} g(x, 0)=0$ for all $x \in \Omega$;

$\overline{\left(g_{2}\right)} \exists p \in\left(2,2^{*}\right)$ such that $\left|g_{t}^{\prime}(x, t)\right| \leq C\left(1+|t|^{p-2}\right)$ for all $x \in \Omega, t \in \mathbb{R}$;

$\overline{\left(g_{3}\right)} g_{t}^{\prime}(x, t)>g(x, t) / t$ for all $x \in \Omega, t \neq 0$;

$\overline{\left(g_{4}\right)} \exists R>0$ and $\theta>2$ such that $0<\theta G(x, t) \leq t g(x, t)$ for all $x \in \Omega,|t| \geq R$, where $G(x, t)=\int_{0}^{t} g(x, s) \mathrm{d} s$.

Under these assumptions, they prove that problem (1.1) has one changing sign solution with exactly two nodal domains and Morse index 2, provided the second eigenvalue of $-\Delta-g_{t}^{\prime}(x, 0)$ is strictly positive. Note that in the case of problem (1.2) it means $\lambda<\lambda_{2}$. Under similar assumptions, in [10] the existence of one solution to problem (1.1) which changes sign exactly once and has connected nodal domains, is proved.

Moreover, in [7] the authors consider a problem similar to (1.2), but with $\lambda<0$; this means that such a problem is substantially similar to problem (1.1), since $-\Delta-\lambda$ induces a positive definite quadratic form whenever $\lambda<\lambda_{1}$. In this case they deal with an autonomous function $g=g(t)$ and they make assumptions analogous to $\overline{\left(g_{1}\right)}-\overline{\left(g_{4}\right)}$ for the autonomous case, with $R=0$ in $\overline{\left(g_{4}\right)}$, proving the existence of three nodal solutions, but with the further assumption that $\Omega$ is big enough.

Finally, in [4], several existence results are given for problem (1.1) with an autonomous $g$, according to the assumptions made on $g, g^{\prime}$ and the growth conditions at infinity (superlinear or asymptotically linear). Concerning the superlinear case, they results can be stated in the following way: if $g$ is of class $C^{1}$ with $g(0)=0, \lambda_{i}<g^{\prime}(0)<\lambda_{i+1}, i \geq 1$, if sign changing solutions are isolated, then there exists a sign changing solution to problem (1.1).

Therefore, Theorem 2.1 in Section 2 can be considered as a counterpart of the results stated above. In fact, no regularity assumption is made on the nonautonomous function $g$ (so no condition is made on its derivative), no restrictions are made on $\Omega$, a multiplicity result is always given and $\lambda$ can be as big as desired, provided it is close to an eigenvalue of $-\Delta$. The novelty of Theorem 2.1 with respect to the results of [19], is that here we give a precise behaviour of the solutions $u_{\lambda}$ for $\lambda \sim \lambda_{i}$, showing that such solutions change sign and giving some properties of their nodal lines (see Ths. 2.1 and 2.2 and their corollaries).

It is also worth mentioning the problem (in the entire space)

$$
\left\{\begin{array}{l}
-\Delta u+a(x) u=g(x, u) \quad \text { in } \mathbb{R}^{N} \\
u \in H^{1}\left(\mathbb{R}^{N}\right)
\end{array}\right.
$$

studied in [5]. Therein, the existence of one sign changing solutions is proved when $g$ is subcritical and superlinear and $a$ satisfies some suitable assumptions. In addition, if $g$ is odd in $u$, the existence of a sequence $u_{k}$ of nodal solutions with at most $k+1$ nodal regions is proved.

Concerning symmetry properties of solutions of nonlinear subcritical homogeneous boundary problems, depending on the geometry of $\Omega$, there are plenty of results. But in such cases the authors assume to deal with positive solutions in presence of functions $g$ 's of class $C^{1}$ (for example, see $[12,16,20]$ and the references quoted therein). For example, in [12] the authors consider the problem

$$
\begin{cases}-\Delta u-\lambda u=g(u) & \text { in } \Omega, \\ u>0 & \text { in } \Omega, \\ u=0 & \text { on } \partial \Omega\end{cases}
$$

where $\Omega$ is convex in the $x_{i}$-direction, symmetric w.r.t. the hyperplane $x_{1}=0$ and $g(0) \geq 0$; using the maximum principle they show that any solution $v$ in $H_{0}^{1}(\Omega)$ of the linearized problem $-\Delta v-\lambda v=\overline{g^{\prime}}(u) v$ is even-symmetric 
in $x_{1}$. In [20] the author considers the problem

$$
\begin{cases}-\Delta u=g(x, u) & \text { in } \Omega, \\ u=0 & \text { on } \partial \Omega,\end{cases}
$$

where $g$ is even in $x_{1}$ and convex in $u$, and she shows that any positive solution $u$ is even in $x_{i}$. Moreover, she also proves that, if $\Omega$ is an annulus or a ball, $g(|x|, \cdot)$ is strictly convex, $u$ is a solution of index 1 , then $u$ is axially symmetric. The author underlines the fact that such a result is not easily applicable to changing sign solutions, since changing sign solutions generally have Morse index greater than 1. Regardless of the Morse index, in Theorem 2.2 we will prove such a symmetry result for the solutions under investigation of problem (1.2).

Of course, more general problems can be considered. For example, in [16] it is shown that solutions $u$ of the problem

$$
\begin{cases}-\Delta u=g(u) & \text { in } \Omega, \\ u>0 & \text { in } \Omega, \\ u=0 & \text { on } \partial \Omega,\end{cases}
$$

where $\Omega$ is a small perturbation of a symmetric domain for which the Gidas, Ni and Nirenberg symmetry result holds, and $g$ is subcritical or critical, are even-symmetric.

The most natural generalization of the previous problems is obtained in presence of the $p$-Laplace operator, when the typical problem is the following:

$$
\begin{cases}-\Delta_{p} u=g(u) & \text { in } \Omega, \\ u>0 & \text { in } \Omega, \\ u=0 & \text { on } \partial \Omega .\end{cases}
$$

Under suitable assumptions on $g$ (typically $g$ of class $C^{1}$, convex, and symmetric in the direction $\nu$ ), symmetry results of the type

$$
\Omega \text { symmetric w.r.t. a direction } \nu \Longrightarrow u \text { is even in the direction } \nu
$$

are given, for example, in $[13,14]$. In this sense our results complement the results given in the papers quoted above.

Another aspect of this paper is a contribution to a nonlinear version of Courant's nodal theorem. More precisely, our result is in the spirit of [11], in which the author proves the following: if $\Omega$ is a bounded (possibly nonsmooth) domain of $\mathbb{R}^{N}$ which is convex and symmetric w.r.t. $k$ orthogonal directions, $1 \leq k \leq N$, then the nodal lines of the eigenfunctions $e_{2}, \ldots, e_{k+1}$ of the problem

$$
\begin{cases}-\Delta u=\lambda u & \text { in } \Omega, \\ u=0 & \text { on } \partial \Omega,\end{cases}
$$

intersect the boundary. In Theorem 2.1, only assuming $\Omega$ smooth enough, we prove that the sign changing solutions found when $\lambda$ belongs to a suitable left neighborhood of $\lambda_{i}$, behave like the eigenfunction $e_{i}$. Therefore, for example, if $i=2$ and $\Omega$ is convex, in Corollary 2.1, we prove that the solutions have exactly two nodal domains and the nodal line touches the boundary of $\Omega$.

For completeness, we also recall a version of Courant's nodal theorem for the $p$-Laplacian found in [15], and that in the case of sublinear elliptic problems in $\mathbb{R}^{N}$, in [2] the authors prove the existence of radial compactly supported solutions with any given number of nodes.

Finally, in Theorem 2.2, we show a result of symmetry in the spirit of (1.3), showing that both the solutions under considerations, i.e. for $\lambda$ close to $\lambda_{i}$, have the same symmetry as $\Omega$. 


\section{Assumptions and main Results}

Throughout this paper, we will consider a bounded domain $\Omega \subset \mathbb{R}^{N}$ which is so smooth that the classical regularity results for solutions of elliptic equations can be applied. To this purpose it is enough to assume, for example, that $\partial \Omega$ is of class $C^{2}$.

Moreover, we will assume some usual conditions for a standard superlinear and subcritical nonlinearity:

$\left(g_{1}\right) g: \Omega \times \mathbb{R} \longrightarrow \mathbb{R}$ is a Carathéodory's function;

$\left(g_{2}\right)$ there exist constants $a>0, s>1$ (and $s<\frac{N+2}{N-2}$ if $N \geq 3$ ) such that $\forall t \in \mathbb{R}$ and for a.e. $x \in \Omega$

$$
|g(x, t)| \leq a|t|^{s}
$$

$\left(g_{3}\right) \forall t \neq 0$ and for a.e. $x$ in $\Omega$

where $\mu=s+1$ and $G(x, t)=\int_{0}^{t} g(x, \sigma) \mathrm{d} \sigma ;$

$$
0<\mu G(x, t) \leq g(x, t) t
$$

$\left(g_{4}\right)$ there exists $c_{1}>0$ such that $G(x, t) \geq c_{1}|t|^{\mu} \forall t \in \mathbb{R}$ and for a.e. $x \in \Omega$.

Such assumptions are quite natural and common when one studies nonlinear subcritical problems (see [1,17,21], the papers quoted above, ...).

Remark 2.1. In order to get existence of nodal solutions, in the papers quoted in the introduction, the authors always assume to deal with a regular function $g$ (at least of class $C^{1}$ ), usually even independent of $x$. Here we only assume $g$ to be continuous in the second variable.

Remark 2.2. We remark that, usually, in $\left(g_{3}\right)$ one requires only $\mu>2$. But by $\left(g_{2}\right)$ and $\left(g_{4}\right)$, one immediately gets $\mu \leq s+1$ (and so $s>1$ ). Therefore a stronger assumption is made on $\mu$; however such an assumption is satisfied whenever $g(x, t)$ "behaves" like $|t|^{s-2} t$.

Remark 2.3. If $g$ is continuous on $\Omega \times \mathbb{R}$, condition $\left(g_{4}\right)$ comes by integration of $\left(g_{3}\right)$, with the constant $c_{1}$ replaced by a function $c_{1}(x)$.

From now on, we will denote by $e_{i}$ any nontrivial element of $E_{\lambda_{i}}$ with $\left\|e_{i}\right\|_{L^{2}}=1$, where $E_{\lambda_{i}}$ denotes the eigenspace of $-\Delta$ on $H_{0}^{1}(\Omega)$ associated to the eigenvalue $\lambda_{i}$. Since $\Omega$ is of class $C^{2}$, it is a classical fact that $e_{i}$ is smooth.

We are now able to state the first result of this paper.

Theorem 2.1. Under hypotheses $\left(g_{1}\right)-\left(g_{4}\right)$, for any $i \geq 2$ there exists $\tau_{i}>0$ such that for any $\lambda \in\left(\lambda_{i}-\tau_{i}, \lambda_{i}\right)$, problem (1.2) has at least two distinct continuous nontrivial sign-changing solutions $u_{\lambda}^{1}$ and $u_{\lambda}^{2}$ such that, for any $k=1,2$,

(a) $u_{\lambda}^{k} \rightarrow 0$ in $H_{0}^{1}(\Omega)$ as $\lambda \rightarrow \lambda_{i}^{-}$;

(b) for any sequence $\left(\mu_{n}\right)_{n} \subset\left(\lambda_{i}-\tau_{i}, \lambda_{i}\right)$ converging to $\lambda_{i}$, the sequence $\left(\frac{u_{\mu_{n}}^{k}}{\left\|u_{\mu_{n}}^{k}\right\|}\right)_{n}$ is compact in $H_{0}^{1}(\Omega)$ and the set of its limits points is contained in the set $\left\{e \in E_{\lambda_{i}}:\|e\|=\frac{1}{\sqrt{\lambda_{i}}}\right\}$;

(c) $\exists C=C(i)>0$ such that $\left\|u_{\lambda}^{k}\right\|_{\infty} \leq C\left\|u_{\lambda}^{k}\right\|$.

Here $\|u\|$ denotes the norm of $u$ in $H_{0}^{1}(\Omega)$, i.e. the $L^{2}$-norm of $D u$.

Remark 2.4. A straightforward corollary of (a) and (b) of Theorem 2.1 is that $u_{\lambda}^{k} \rightarrow 0$ uniformly in $\Omega$ as $\lambda \rightarrow \lambda_{i}^{-}$for any $k=1,2$.

A remarkable consequence of the previous theorem is the following nonlinear version of Courant's Nodal theorem.

Corollary 2.1. Assume that $\Omega \subset \mathbb{R}^{N}, N \geq 2$, is such that the Courant's nodal theorem holds, that $g: \Omega \times \mathbb{R} \longrightarrow$ $\mathbb{R}$ is of class $C^{1}$ and that $t^{2} \frac{\partial g}{\partial t}(x, t)>\operatorname{tg}(x, t)$ for all $(x, t) \in \Omega \times \mathbb{R}$. Moreover, assume that $\lambda_{2}$ is simple and that $\left(g_{1}\right)-\left(g_{4}\right)$ hold. Then the solutions $u_{\lambda}$ 's found in Theorem 2.1 for $i=2$ have exactly two nodal domains and the nodal line of each $u_{\lambda}$ intersects $\partial \Omega$. 
Remark 2.5. According to Theorem 1.1 in [11], the Courant's nodal theorem holds, for example, if $\Omega \subset \mathbb{R}^{N}$ is convex and symmetric w.r.t. $k$ orthogonal directions, $1 \leq k \leq N$.

Proof of Corollary 2.1. Since each eigenvalue of $-\Delta$ has finite multiplicity and $\lambda_{2}$ is simple, we have $\lambda_{1}<\lambda<$ $\lambda_{2}<\lambda_{3}$. Since each $u_{\lambda}$ is found by a linking structure involving the decomposition $H_{0}^{1}(\Omega)=\operatorname{Span}\left(e_{1}\right) \oplus$ $\operatorname{Span}\left(e_{2}\right) \oplus \operatorname{Span}\left(e_{3}, \ldots\right)$ (see [19]), it is a classical fact that $i\left(u_{\lambda}\right) \leq 2$ (see [8]) and \#\{nodal regions $\} \leq i\left(u_{\lambda}\right)$, where $i\left(u_{\lambda}\right)$ denotes the Morse index of $u_{\lambda}$ (it is enough to adapt the proof of [8] under the assumptions on $g_{t}$, similarly to [3]). We remark that, since $u_{\lambda} \in C(\bar{\Omega})$, no further growth assumption on $g_{t}^{\prime}$ are needed. Moreover, since each $u_{\lambda}$ changes sign, for $\frac{u_{\lambda}}{\left\|u_{\lambda}\right\|} \rightarrow t_{0} e_{2}\left(t_{0}^{2}=1 / \lambda_{i}\right)$, we get that each $u_{\lambda}$ has exactly two nodal domains. Let us set $\Omega^{+}=\left\{x \in \Omega: t_{0} e_{2}(x)>0\right\}$.

Let us prove that the nodal line $\Gamma_{\lambda}$ of each $u_{\lambda}$ cannot be closed. In fact, there exists $\varepsilon>0$ such that $\forall \lambda \in\left(\lambda_{i}-\tau_{i}, \lambda_{i}\right)$ and $\forall x \in \Omega^{+}$with $d\left(x, \partial \Omega^{+}\right) \geq \varepsilon$ it holds $u_{\lambda}(x) \geq 0$ and $\forall x \in \Omega \backslash \Omega^{+}$such that $d\left(x, \partial \Omega^{+}\right) \geq \varepsilon$ it holds $u_{\lambda}(x) \leq 0$. Otherwise we could find, for example, a point $x_{\lambda}$ and then, by continuity of $u_{\lambda}$, an open set $A_{\lambda}$ contained in $\Omega^{+}$, where $u_{\lambda}$ is negative and with $x_{\lambda}$ converging to a point $x_{0} \in \partial \Omega$. Of course $\frac{\partial u_{\lambda}}{\partial \nu}\left(x_{0}\right) \geq 0$.

Assume that $\frac{u_{\lambda}}{\left\|u_{\lambda}\right\|} \rightarrow t_{0} e_{2}$ in $W^{2, q}(\Omega)$ for some $q$. By the trace theorem there exists a universal constant $T>0$ such that

$$
\left\|D \frac{u_{\lambda}}{\left\|u_{\lambda}\right\|}-t_{0} D e_{2}\right\|_{L^{q}(\partial \Omega)} \leq T\left\|D \frac{u_{\lambda}}{\left\|u_{\lambda}\right\|}-t_{0} D e_{2}\right\|_{W^{1, q}(\Omega)} \leq T\left\|\frac{u_{\lambda}}{\left\|u_{\lambda}\right\|}-t_{0} e_{2}\right\|_{W^{2, q}(\Omega)} \rightarrow 0 \quad \text { as } \lambda \rightarrow \lambda_{i} .
$$

Therefore we can assume that $D \frac{u_{\lambda}}{\left\|u_{\lambda}\right\|} \rightarrow t_{0} D e_{2}$ a.e. on $\partial \Omega$. In particular we can choose $x_{\lambda} \in A_{\lambda}$ converging to a point $x_{0} \in \partial \Omega$ where $D \frac{u_{\lambda}}{\left\|u_{\lambda}\right\|}$ converges to $t_{0} D e_{2}$. Then $\frac{\partial u_{\lambda}}{\partial \nu}\left(x_{0}\right) \rightarrow \frac{\partial t_{0} e_{2}}{\partial \nu}\left(x_{0}\right)$ and a contradiction arises, since by classical results $\frac{\partial t_{0} e_{2}}{\partial \nu} \mid \partial \Omega^{+}<0$.

Finally, let us show that $\frac{u_{\lambda}}{\left\|u_{\lambda}\right\|} \rightarrow t_{0} e_{2}$ in $W^{2, q}(\Omega)$, where $q$ is chosen so large that $W^{2, q}(\Omega) \hookrightarrow L^{\infty}(\Omega)($ see the following section for more details). First of all, we get that $\frac{u_{\lambda}}{\left\|u_{\lambda}\right\|} \rightarrow t_{0} e_{2}$ in $W^{2, q}(\Omega)$ as $\lambda \rightarrow \lambda_{i}^{-}$. Indeed the function $v_{\lambda}:=\frac{u_{\lambda}}{\left\|u_{\lambda}\right\|}-t_{0} e$ solves the problem

$$
\begin{cases}-\Delta v_{\lambda}=\lambda v_{\lambda}+\left(\lambda-\lambda_{i}\right) t_{0} e_{2}+\frac{g\left(x, u_{\lambda}\right)}{\left\|u_{\lambda}\right\|} & \text { in } \Omega \\ u=0 & \text { on } \partial \Omega\end{cases}
$$

By the regularity inequality of Calderón-Zygmund (see [22], Th. B.2) and by assumption $\left(g_{2}\right)$ we get

$$
\left\|v_{\lambda}\right\|_{2, q} \leq C\left(\lambda_{i}\left\|v_{\lambda}\right\|_{q}+a \frac{\left\|u_{\lambda}\right\|_{q s}^{s}}{\left\|u_{\lambda}\right\|}+\left(\lambda_{i}-\lambda\right)\left\|t_{0} e_{2}\right\|_{q}\right)
$$

for some universal constant $C>0$. By (c) of Theorem $2.1\left\|u_{\lambda}\right\|_{q s}^{s} \leq c_{1}\left\|u_{\lambda}\right\|^{s}$ for some $c_{1}>0$ independent of $\lambda$ and also $\left\|v_{\lambda}\right\|_{q}$ is bounded; therefore also $\left\|v_{\lambda}\right\|_{2, q}$ is bounded. Thus we can assume that $\frac{u_{\lambda}}{\left\|u_{\lambda}\right\|} \rightarrow t_{0} e_{2}$ in $W^{2, q}(\Omega)$. But then $v_{\lambda} \rightarrow 0$ in $W^{1, q}(\Omega)$, so that (2.4) implies that $v_{\lambda} \rightarrow 0$ in $W^{2, q}(\Omega)$.

Concerning the symmetry result, we will specialize the class of nonlinearities, assuming

$\widetilde{\left(g_{1}\right)}: g: \Omega \times \mathbb{R} \longrightarrow \mathbb{R}$ is of class $C^{1}$ and there exist $b \in L^{q}(\Omega)$ and $p \geq 1$ such that

$$
\left|g_{t}(x, t)\right| \leq b(x)|t|^{p} \quad \forall t \in \mathbb{R} \text { and for a.e. } x \in \Omega \text {. }
$$

Here $q \geq 1$ if $N<3$ and $q=N / 2$ if $N \geq 3$.

Theorem 2.2. Let $\Omega$ be a bounded domain of $\mathbb{R}^{2}$ which is convex and symmetric w.r.t. two orthogonal directions, say the $x_{i}$-directions, $i=1,2$. Assume $\widetilde{\left(g_{1}\right)}-\left(g_{2}\right)-\left(g_{3}\right)-\left(g_{4}\right)$ and $g\left(x_{1},-x_{2},-s\right)=-g(x, s)$ for any $s \in \mathbb{R}$ and 
for a.e. $x \in \Omega$. Then there exists $\tau_{2}^{\prime} \leq \tau_{2}$ such that $\forall \lambda \in\left(\lambda_{2}-\tau_{2}^{\prime}, \lambda_{2}\right)$, any solution $u_{\lambda}$ of problem (1.2) found in Theorem 2.1 satisfies $u_{\lambda}(x)=-u_{\lambda}\left(x_{1},-x_{2}\right)$.

\section{Proof of Theorem 2.1}

Let us introduce the $C^{1}$ functional $f_{\lambda}: H_{0}^{1}(\Omega) \longrightarrow \mathbb{R}$ defined by

$$
f_{\lambda}(u)=\frac{1}{2} \int_{\Omega}|D u|^{2} \mathrm{~d} x-\frac{\lambda}{2} \int_{\Omega} u^{2} \mathrm{~d} x-\int_{\Omega} G(x, u) \mathrm{d} x,
$$

whose critical points are the solutions of problem (1.2).

Let us denote by $u_{\lambda}$ one of the two families of solutions to (1.2) found in [19] with the property that $f_{\lambda}\left(u_{\lambda}\right) \rightarrow 0$ as $\lambda \rightarrow \lambda_{i}, i \geq 2$.

The proof of Theorem 2.1 will be given in several steps.

First step. $u_{\lambda} \rightarrow 0$ in $H_{0}^{1}(\Omega)$.

Proof. Since $u_{\lambda}$ solves (1.2), we have

$$
\int_{\Omega} D u_{\lambda} \cdot D v \mathrm{~d} x-\lambda \int_{\Omega} u_{\lambda} v \mathrm{~d} x-\int_{\Omega} g\left(x, u_{\lambda}\right) v \mathrm{~d} x=0 \quad \forall v \in H_{0}^{1}(\Omega),
$$

and in particular

$$
\int_{\Omega}\left|D u_{\lambda}\right|^{2} \mathrm{~d} x-\lambda \int_{\Omega} u_{\lambda}^{2} \mathrm{~d} x-\int_{\Omega} g\left(x, u_{\lambda}\right) u_{\lambda} \mathrm{d} x=0 .
$$

In this way

$$
f_{\lambda}(u)=\int_{\Omega}\left[\frac{1}{2} g\left(x, u_{\lambda}\right) u_{\lambda}-G\left(x, u_{\lambda}\right)\right] \mathrm{d} x \geq\left(\frac{\mu}{2}-1\right) \int_{\Omega} G\left(x, u_{\lambda}\right) \mathrm{d} x>0 .
$$

But $f_{\lambda}\left(u_{\lambda}\right) \rightarrow 0$ as $\lambda \rightarrow \lambda_{i}$, and so $\int G\left(x, u_{\lambda}\right) \rightarrow 0$ as $\lambda \rightarrow \lambda_{i}$, which implies

$$
u_{\lambda} \longrightarrow 0 \quad \text { in } L^{\mu}(\Omega)
$$

by $\left(g_{4}\right)$. Therefore, also $u_{\lambda} \rightarrow 0$ strongly in $H_{0}^{1}(\Omega)$. In fact

$$
f_{\lambda}\left(u_{\lambda}\right)=\frac{1}{2} \int_{\Omega}\left|D u_{\lambda}\right|^{2} \mathrm{~d} x-\frac{1}{2} \lambda \int_{\Omega} u_{\lambda}^{2} \mathrm{~d} x-\int_{\Omega} G\left(x, u_{\lambda}\right) u_{\lambda} \mathrm{d} x,
$$

and passing to the limit we get

$$
\left\|u_{\lambda}\right\| \rightarrow 0
$$

We can now assume that there is $u \in H_{0}^{1}(\Omega)$ such that $u_{\lambda} /\left\|u_{\lambda}\right\| \rightarrow u$ in $H_{0}^{1}(\Omega)$.

Second step. $u$ solves $-\Delta u=\lambda_{i} u$ in $H_{0}^{1}(\Omega)$.

Proof. If $v \in H_{0}^{1}(\Omega),(3.5)$ gives

$$
\int_{\Omega} \frac{D u_{\lambda}}{\left\|u_{\lambda}\right\|} \cdot D v \mathrm{~d} x-\lambda \int_{\Omega} \frac{u_{\lambda}}{\left\|u_{\lambda}\right\|} v \mathrm{~d} x=\int_{\Omega} \frac{g\left(x, u_{\lambda}\right)}{\left\|u_{\lambda}\right\|} v \mathrm{~d} x .
$$


The growth assumption $\left(g_{2}\right)$, Hölder and Sobolev inequalities imply

$$
\left|\int_{\Omega} \frac{g\left(x, u_{\lambda}\right)}{\left\|u_{\lambda}\right\|} v \mathrm{~d} x\right| \leq a \int_{\Omega} \frac{\left|u_{\lambda}\right|^{s}}{\left\|u_{\lambda}\right\|}|v| \mathrm{d} x \leq c\left\|u_{\lambda}\right\|^{s-1}\|v\|_{L^{\frac{2^{*}}{2^{*}-s}}},
$$

where $c>0$ is independent of $u_{\lambda}$.

In this way, passing to the limit in (3.8),

$$
\int_{\Omega} D u \cdot D v \mathrm{~d} x=\lambda_{i} \int_{\Omega} u v \mathrm{~d} x \quad \forall v \in H_{0}^{1}(\Omega),
$$

i.e. $u$ solves $-\Delta u=\lambda_{i} u$ in $H_{0}^{1}(\Omega)$, and so there exists $t_{0} \in \mathbb{R}$ such that $u=t_{0} e_{i}$, where $e_{i} \in E_{\lambda_{i}}$ (see Th. 2.1).

Third step. $u_{\lambda} /\left\|u_{\lambda}\right\| \rightarrow t_{0} e_{i}$ strongly in $H_{0}^{1}(\Omega)$, as $\lambda \rightarrow \lambda_{i}$, where $t_{0}^{2}=\frac{1}{\lambda_{i}}$.

Proof. Equation (3.6) and $\left(g_{2}\right)$ imply that

$$
1=\lim _{\lambda \rightarrow \lambda_{i}} \int_{\Omega} \frac{\left|D u_{\lambda}\right|^{2}}{\left\|u_{\lambda}\right\|^{2}} \mathrm{~d} x=\lambda_{i} \int_{\Omega} u^{2} \mathrm{~d} x
$$

In fact,

$$
\left|\int_{\Omega} \frac{g\left(x, u_{\lambda}\right)}{\left\|u_{\lambda}\right\|} u_{\lambda} \mathrm{d} x\right| \leq a \int_{\Omega} \frac{\left|u_{\lambda}\right|^{s+1}}{\left\|u_{\lambda}\right\|} \mathrm{d} x \leq c_{1}\left\|u_{\lambda}\right\|^{s-1}
$$

by Hölder and Sobolev inequalities, $c_{1}=c_{1}(\Omega, N)>0$ being independent of $u_{\lambda}$.

But equation (3.9) implies that

$$
\lambda_{i} \int_{\Omega} u^{2} \mathrm{~d} x=\int_{\Omega}|D u|^{2} \mathrm{~d} x
$$

so that $\|u\|=1$ and then the convergence is strong.

The last equation gives the possible values of $t_{0}$.

Now, let us prove the following preliminary result.

Proposition 3.1. Assume $N \leq 5$ and one of the following hypotheses:

- $s<4$ if $N=3$;

- $s<2$ if $N=4$;

- $s<\frac{4}{3}$ if $N=5$.

Then $u_{\lambda} \in C(\bar{\Omega})$ and there exist $\tau_{i}>0$ and $C=C(\Omega, N, i)>0$ such that $\forall \lambda \in\left(\lambda_{i}-\tau_{i}, \lambda_{i}\right)$

$$
\left\|u_{\lambda}\right\|_{\infty} \leq C\left\|u_{\lambda}\right\|
$$

Proof. Since $\Omega$ is of class $C^{2}$, it is a classical fact (see for example [22], Lem. B.3 and below) that any solution $u$ of $(1.2)$ belongs to $C(\bar{\Omega})$. In order to get the $L^{\infty}-H_{0}^{1}$ estimate, let us note that $u \in W^{2, q}(\Omega)$, provided $\lambda u+g(x, u) \in L^{q}(\Omega)$, and there exists $C>0$ such that

$$
\|u\|_{W^{2, q}} \leq C\|\lambda u+g(x, u)\|_{L^{q}} .
$$

Of course $\left(g_{2}\right)$ implies that $\lambda u+g(\cdot, u) \in L^{q}(\Omega)$ if $\lambda|u|+a|u|^{s} \in L^{q}(\Omega)$. Moreover

$$
\left\|\lambda u+a|u|^{s}\right\|_{q} \leq \lambda\|u\|_{q}+\left\||u|^{s}\right\|_{q}=\lambda\|u\|_{q}+\|u\|_{q s}^{s}
$$


and by Sobolev inequality

$$
\leq \gamma\left(\|u\|+\|u\|^{s}\right)
$$

provided $q s \leq 2^{*}$, and $\gamma>0$ is a constant depending on $\Omega, N, q$ and $i$.

Since $\left\|u_{\lambda}\right\| \rightarrow 0$ as $\lambda \rightarrow \lambda_{i}$, there exists $\tau_{i}>0$ such that $\left\|u_{\lambda}\right\| \leq 1 \forall \lambda \in\left(\lambda_{i}-\tau_{i}, \lambda_{i}\right)$, so that for such $\lambda$ 's $\left\|u_{\lambda}\right\|^{s} \leq\left\|u_{\lambda}\right\|$. Therefore, (3.10) and the estimate above imply the existence of a constant $C_{1}=C_{1}(\Omega, N, q, i)>0$ such that

If

$$
\left\|u_{\lambda}\right\|_{W^{2, q}} \leq C\left\|u_{\lambda}\right\| \quad \forall \lambda \in\left(\lambda_{i}-\tau_{i}, \lambda_{i}\right)
$$

Morrey's embedding theorem guarantees that

$$
\frac{1}{q}-\frac{2}{N}<0
$$

$$
\left\|u_{\lambda}\right\|_{\infty} \leq C_{2}\left\|u_{\lambda}\right\|_{W^{2, q}}
$$

where $C_{2}$ is a universal constant.

Therefore we require $q>\frac{N}{2}$ in order to get

$$
\left\|u_{\lambda}\right\|_{\infty} \leq C_{3}\left\|u_{\lambda}\right\|
$$

for some $C_{3}>0$ depending on $\Omega, N, q$ and $i$.

Of course, if $N=1$ or $N=2$, it is clear that any $q$ large enough can be found.

If $N=3$ one has to choose $\frac{3}{2}<q \leq \frac{6}{s}$, which is solvable only if $s<4$.

If $N=4$ one has to choose $2<q \leq \frac{4}{s}$, which is solvable only if $s<2$.

If $N=5$ one has to choose $\frac{5}{2}<q \leq \frac{10}{s}$, which is solvable only if $s<\frac{4}{3}$.

If $N \geq 6$ it is clear that the system $\frac{N}{2}<q \leq \frac{2^{*}}{s}$ cannot be solved.

However, some refinements of the considerations above can be done invoking Moser's iteration technique (see [18]) and its development due to Brezis and Kato (see [9]). Therefore we now generalize Proposition 3.1 to get the following general $L^{\infty}-H_{0}^{1}$ estimate, after remarking again that $u_{\lambda} \in C(\bar{\Omega})$. In this way Theorem 2.1 will be completely proved.

Proposition 3.2. Under the assumptions of Theorem 2.1, there exist $\tau_{i}>0$ and $C=C(\Omega, N, i)>0$ such that $\forall \lambda \in\left(\lambda_{i}-\tau_{i}, \lambda_{i}\right), u_{\lambda} \in C(\bar{\Omega})$ and

$$
\left\|u_{\lambda}\right\|_{\infty} \leq C\left\|u_{\lambda}\right\|
$$

Proof. Let us rewrite the equation $-\Delta u-\lambda u=g(x, u)$ as

$$
-\Delta u=\alpha(x)(1+|u|)
$$

where

$$
\alpha(x):=\frac{\lambda u(x)+g(x, u(x))}{1+|u(x)|} .
$$

By standard regularity results, any solution in $H_{0}^{1}(\Omega)$ of $(3.11)$ belongs to $L^{q}(\Omega)$ for any $q \in[1, \infty)$. Such a result is based on the classical iteration technique

$$
\text { if } u \in L^{2\left(q_{j-1}+1\right)}(\Omega) \Rightarrow u \in L^{2\left(q_{j}+1\right)}(\Omega) \text {, }
$$

where

$$
q_{j}+1=\left(q_{j-1}+1\right) \frac{N}{N-2}, \quad j \geq 1 \quad \text { and } q_{0}=0
$$


which provides the following estimate for any $q_{j}<\infty$ (for example, see [22], Lem. B.3):

$$
\left.\left.\int_{\Omega}|D| u\right|^{q_{j}+1}\right|^{2} \mathrm{~d} x \leq \frac{C_{q_{j}}\left(1+c_{*}\right)}{\frac{1}{2}-\frac{q_{j}^{2}}{4\left(q_{j}+1\right)^{2}}},
$$

where

and $c_{*} \geq 0$ is such that

$$
C_{q_{j}}=\max \left\{\|\alpha\|_{\frac{N}{2}}|\Omega|^{\frac{N}{N-2}}, 3\|u\|_{2 q_{j}+2}^{2 q_{j}+2}\right\}
$$

$$
\int_{\left\{|\alpha|>c_{*}\right\}}|\alpha|^{N / 2} \mathrm{~d} x \leq \frac{1}{24}
$$

More precisely, one proves that $|u|^{q_{j-1}+1} \in H_{0}^{1}(\Omega) \hookrightarrow L^{2^{*}}(\Omega)$, and then $u \in L^{\frac{2 N\left(q_{j-1}+1\right)}{N-2}}(\Omega) \forall j$. This implies that $u \in L^{q}(\Omega) \forall q<\infty$. In this way, by the Calderón-Zygmund inequality (see [22], Th. B.2), $u \in W^{2, q}(\Omega)$ $\forall q<\infty$ and

$$
\|u\|_{W^{2, q}} \leq C(q)\|\lambda u+g(\cdot, u)\|_{q} .
$$

If $q$ is sufficiently large $(2 q>N), W^{2, q}(\Omega) \hookrightarrow C(\bar{\Omega})$ and there exists $\tilde{C}_{q}>0$ such that

$$
\|v\|_{\infty} \leq \tilde{C_{q}}\|v\|_{W^{2, q}} \quad \forall v \in W^{2, q}(\Omega) .
$$

Thus, by (3.15) and (3.16), there exists $C_{1}(q)>0$ such that

$$
\|u\|_{\infty} \leq C_{1}(q)\|\lambda u+g(\cdot, u)\|_{q} \leq C_{1}(q)\left(\lambda_{i}\|u\|_{q}+a\|u\|_{q s}^{s}\right) .
$$

If $q s \leq 2^{*}$, then $\|u\|_{q},\|u\|_{q s} \leq C\|u\|$, where $C$ is independent of $u$. But $\|u\| \rightarrow 0$ as $\lambda \rightarrow \lambda_{i}$, so that there exists $\tau_{i}>0$ such that $\|u\|^{s} \leq\|u\|$ for any $\lambda \in\left(\lambda_{i}-\tau_{i}, \lambda_{i}\right)$ and from (3.17) the Proposition is proved (this is essentially the case of Prop. 3.1).

If $q s>2^{*}$, first we observe that $\|u\|_{q} \leq C(\Omega)\|u\|_{q s}, C(\Omega)$ being independent of $u$, so that it will be enough to estimate $\|u\|_{q s}$ in terms of $\|u\|$.

First of all, note that if $u \in H_{0}^{1}(\Omega) \cap L^{\infty}(\Omega)$ and $r>2^{*}$, then

$$
\int_{\Omega}|u|^{r} \mathrm{~d} x \leq\|u\|_{\infty}^{r-2^{*}}\|u\|_{2^{*}}^{2^{*}} \leq \gamma\|u\|_{\infty}^{r-2^{*}}\|u\|^{2^{*}}
$$

by Sobolev theorem, so that

where $C_{1}>0$ is independent of $u$.

$$
\|u\|_{r} \leq C_{1}\|u\|_{\infty}^{1-\frac{2^{*}}{r}}\|u\|^{2^{*}}
$$

Therefore, (3.17) and (3.18) imply

$$
\|u\|_{\infty} \leq C_{i}\left(\|u\|_{q s}+\|u\|_{q s}^{s}\right) \leq C_{i}^{\prime}\left(\|u\|_{\infty}^{1-\frac{2^{*}}{q s}}\|u\|^{\frac{2^{*}}{q s}}+\|u\|_{\infty}^{s\left(1-\frac{2^{*}}{q s}\right)}\|u\|^{\frac{2^{*}}{q}}\right)
$$

where $C_{i}, C_{i}^{\prime}>0$ are independent of $u$, but depend on $i$.

If one proves that

then

$$
\left\|u_{\lambda}\right\|_{\infty} \rightarrow 0 \quad \text { as } \lambda \rightarrow \lambda_{i}^{-},
$$

$$
\left\|u_{\lambda}\right\|_{\infty}^{s\left(1-\frac{2^{*}}{q s}\right)} \leq\left\|u_{\lambda}\right\|_{\infty}^{1-\frac{2^{*}}{q s}}
$$

Moreover, $\left\|u_{\lambda}\right\| \rightarrow 0$, and then $\left\|u_{\lambda}\right\|^{\frac{2^{*}}{q}} \leq\left\|u_{\lambda}\right\|^{\frac{2^{*}}{q s}}$ for every $\lambda$ in a suitable left neighborhood of $\lambda_{i}$, say $\left(\lambda_{i}-\tau_{i}, \lambda_{i}\right)$. 
In this way (3.19) implies

$$
\left\|u_{\lambda}\right\|_{\infty} \leq C_{i}^{\prime}\left(\left\|u_{\lambda}\right\|_{\infty}^{1-\frac{2^{*}}{q s}}\left\|u_{\lambda}\right\|^{\frac{2^{*}}{q s}}+\left\|u_{\lambda}\right\|_{\infty}^{1-\frac{2^{*}}{q s}}\left\|u_{\lambda}\right\|^{\frac{2^{*}}{q s}}\right)
$$

that is

$$
\left\|u_{\lambda}\right\|_{\infty}^{\frac{2^{*}}{q s}} \leq C_{i}^{\prime \prime}\left\|u_{\lambda}\right\|^{\frac{2^{*}}{q s}}
$$

for some $C_{i}^{\prime \prime}>0$ independent of $u_{\lambda}$, so that the thesis follows.

Therefore, in order to conclude, we only need to prove (3.20). Its proof consists in a standard bootstrap argument based on inequality (3.13). This leads to an estimate which is less interesting than the one stated in the Proposition, but is enough to prove the main result. In fact, such a bootstrap argument gives an inequality of the form

$$
\left\|u_{\lambda}\right\|_{\infty} \leq C\left\|u_{\lambda}\right\|^{\delta}
$$

for some $C>0$ and $\delta \in(0,1)$ independent of $u$ and for any $\lambda \in\left(\lambda_{i}-\tau_{i}, \lambda_{i}\right)$, and this is enough to prove (3.20) thanks to (3.7). The proof of (3.21) is classical and we only sketch it for the sake of completeness.

From (3.17) it is enough to estimate $\left\|u_{\lambda}\right\|_{q}$ and $\left\|u_{\lambda}\right\|_{q s}$ in terms of $\left\|u_{\lambda}\right\|$. To this aim, take $q$ so large that $q s>N$, so that $W_{0}^{1, q s}(\Omega) \hookrightarrow C(\bar{\Omega})$ and there exists $c=c(N, q, s)>0$ such that

$$
\|v\|_{\infty} \leq c\|D v\|_{q s} \quad \text { for every } v \in W_{0}^{1, q s}(\Omega) .
$$

Let $j \in \mathbb{N}$ be such that $q s \leq \frac{2 N\left(q_{j}+1\right)}{N-2}$, where $q_{j}$ is as in (3.12) and (3.13). In this way, we will apply (3.22) to the function $|u|^{q_{j}+1}$, and from (3.13) we get

$$
\|u\|_{\infty} \leq c_{1}\left(\frac{C_{q_{j}}\left(1+c_{*}\right)}{\frac{1}{2}-\frac{q_{j}^{2}}{4\left(q_{j}+1\right)^{2}}}\right)^{\frac{1}{2 q_{j}+2}}
$$

for some $c_{1}=c_{1}(N, q, s)>0$. Thus, to get (3.21), in view of (3.14) it is enough to estimate $\|\alpha\|_{\frac{N}{2}}$ and $\|u\|_{2 q_{j}+2}$ in terms of powers of $\|u\|$.

First observe that

$$
\|\alpha\|_{\frac{N}{2}} \leq \lambda_{i}\left\|\frac{u}{1+|u|}\right\|_{\frac{N}{2}}+a\left\||u|^{s-1}\right\|_{\frac{N}{2}} .
$$

Since $s<2^{*}-1$, we get $(s-1) \frac{N}{2}<2^{*}$ and by Sobolev inequality

$$
\left\||u|^{s-1}\right\|_{\frac{N}{2}} \leq \gamma\|u\|^{s-1}
$$

for some universal constant $\gamma$.

As for $\left\|\frac{u}{1+|u|}\right\|_{\frac{N}{2}}$, if $N \leq 6$ we have

$$
\int_{\Omega}\left(\frac{|u|}{1+|u|}\right)^{N / 2} \mathrm{~d} x \leq \int_{\Omega}|u|^{N / 2} \mathrm{~d} x
$$

and $N / 2 \leq 2^{*}$, so that Sobolev inequality gives $\left\|\frac{u}{1+|u|}\right\|_{\frac{N}{2}} \leq \gamma^{\prime}\|u\|$. In the case $N>6$, note that for any $\varepsilon \in(0,1)$, it holds

$$
1+|u| \geq \frac{1}{\varepsilon^{\varepsilon}(1-\varepsilon)^{1-\varepsilon}}|u|^{\varepsilon}=C_{\varepsilon}^{-1}|u|^{\varepsilon},
$$


where we have set

$$
C_{\varepsilon}=\varepsilon^{\varepsilon}(1-\varepsilon)^{1-\varepsilon}
$$

Since $N \geq 7$, one can take

$$
\varepsilon \in\left[1-\frac{4}{N-2}, 1-\frac{2}{N}\right]
$$

so that

$$
\int_{\Omega}\left(\frac{|u|}{1+|u|}\right)^{N / 2} \mathrm{~d} x \leq C_{\varepsilon}^{-1} \int_{\Omega}|u|^{(1-\varepsilon) N / 2} \mathrm{~d} x .
$$

By (3.25),

$$
1 \leq(1-\varepsilon) N / 2 \leq 2^{*}
$$

and by Sobolev inequality

$$
\int_{\Omega}\left(\frac{|u|}{1+|u|}\right)^{N / 2} \mathrm{~d} x \leq \bar{C}\|u\|^{(1-\varepsilon) N / 2}
$$

for some $\bar{C}>0$ independent of $u$.

Finally, (3.23), (3.24) and (3.26) imply the existence of a constant $M=M\left(\Omega, \lambda_{i}\right)>0$ independent of $u$ such that

$$
\|\alpha\|_{\frac{N}{2}} \leq M\left(\|u\|^{s-1}+\|u\|+\|u\|^{(1-\varepsilon) N / 2}\right) .
$$

Concerning the norm $\|u\|_{2 q_{j}+2}$ appearing in (3.14), if $2 q_{j}+2 \leq 2^{*}$ it can be estimated directly by $\|u\|$. If $2 q_{j}+2>2^{*}$, we can rewrite the analogous of (3.13) for $q_{j-1}+1$, that is

$$
\left.\left.\int_{\Omega}|D| u\right|^{q_{j-1}+1}\right|^{2} \mathrm{~d} x \leq \frac{C_{q_{j-1}}\left(1+c_{*}\right)}{\frac{1}{2}-\frac{q_{j-1}^{2}}{4\left(q_{j-1}+1\right)^{2}}},
$$

so that by Sobolev inequality and (3.12), we can estimate $\|u\|_{2 q_{j}+2}$ in terms of the left hand side of (3.27). If $q_{j-1}+1 \leq 2^{*}$ we conclude. Otherwise, after a finite number of steps we are reduced to estimate $u_{q_{j-k}+1}(\Omega)$ in terms of powers of $\|u\|$ for some $k \in \mathbb{N}$ and the usual bootstrap argument applies.

Finally, since $\left\|u_{\lambda}\right\| \leq 1 \forall \lambda \in\left(\lambda_{i}-\tau_{i}, \lambda_{i}\right)$, (3.21) follows.

\section{Proof of Theorem 2.2}

In this section, we will always assume assumptions $\widetilde{\left(g_{1}\right)},\left(g_{2}\right),\left(g_{3}\right)$ and $\left(g_{4}\right)$.

Lemma 4.1. Assume that $\Omega$ is symmetric w.r.t. the $j$-th axis, $1 \leq j \leq N$, that $g\left(x_{1}, \ldots,-x_{j}, \ldots, x_{n},-s\right)=$ $-g(x, s)$, and that $u$ solves (1.2). Then also $\bar{u}(x):=-u\left(x_{1}, \ldots,-x_{j}, \ldots, x_{n}\right)$ solves (1.2).

Note that such a symmetry on $g$ leads to the existence of infinitely many solutions to problem (1.2) (see, for example, [21]).

Proof. Let $\varphi \in H_{0}^{1}(\Omega)$. Then

$$
\begin{aligned}
\int_{\Omega} D \bar{u} \cdot D \varphi \mathrm{d} x-\int_{\Omega}[\lambda \bar{u}-g(x, \bar{u})] \varphi \mathrm{d} x \quad\left(\text { change } x_{j} \mapsto-x_{j}\right) & =\int_{\Omega} D u \cdot D \varphi\left(x_{1}, \ldots,-x_{j}, \ldots, x_{n}\right) \mathrm{d} x \\
& -\int_{\Omega}[\lambda u-g(x, u)] \varphi\left(x_{1}, \ldots,-x_{j}, \ldots, x_{n}\right) \mathrm{d} x=0
\end{aligned}
$$

since $u$ is a solution of (1.2). 
Now set

$$
U_{\lambda}(x):=u_{\lambda}(x)-\bar{u}_{\lambda}(x) .
$$

We will prove Theorem 2.2 by proving the following final

Lemma 4.2. Under the assumptions of Lemma 4.1, if every element in $E_{\lambda_{i}}$ (see Sect. 2) has only one nodal line, which is not closed, then there exists $\tau_{i}^{\prime} \in\left(0, \tau_{i}\right]$ such that $U_{\lambda} \equiv 0$ for any $\lambda \in\left(\lambda_{i}-\tau_{i}^{\prime}, \lambda_{i}\right)$.

Remark 4.1. The assumption "every element in $E_{\lambda_{i}}$ has only one nodal line, which is not closed" is of course satisfied when $i=2$ and the Courant Nodal theorem holds.

Proof of Lemma 4.2. Assume by contradiction that $U_{\lambda} \not \equiv 0$. Of course $U_{\lambda} \rightarrow 0$ in $H_{0}^{1}(\Omega)$ and uniformly as a consequence of (3.7) and of Proposition 3.2. Now, we prove that, along sequences, $U_{\lambda} /\left\|U_{\lambda}\right\| \rightarrow \pm \frac{1}{\sqrt{\lambda_{i}}} e_{i}$. Indeed we can assume that $U_{\lambda} /\left\|U_{\lambda}\right\| \rightarrow U$ in $H_{0}^{1}(\Omega)$ and a.e. in $\Omega$ as $\lambda \rightarrow \lambda_{i}$. By Lemma 4.1, $U_{\lambda}$ solves

$$
\begin{cases}-\Delta U_{\lambda}-\lambda U_{\lambda}=g\left(x, u_{\lambda}\right)-g\left(x, \bar{u}_{\lambda}\right) & \text { in } \Omega \\ U_{\lambda}=0 & \text { on } \partial \Omega .\end{cases}
$$

Thus, if $\varphi \in H_{0}^{1}(\Omega)$,

$$
\int_{\Omega} \frac{D U_{\lambda}}{\left\|U_{\lambda}\right\|} \cdot D \varphi \mathrm{d} x-\lambda \int_{\Omega} \frac{U_{\lambda}}{\left\|U_{\lambda}\right\|} \varphi \mathrm{d} x=\int_{\Omega} \frac{g\left(x, u_{\lambda}\right)-g\left(x, \bar{u}_{\lambda}\right)}{\left\|U_{\lambda}\right\|} \varphi \mathrm{d} x .
$$

By the Mean Value theorem, there exists $v_{\lambda}$ with values between $u_{\lambda}$ and $\bar{u}_{\lambda}$ such that

$$
\left|g\left(x, u_{\lambda}\right)-g\left(x, \bar{u}_{\lambda}\right)\right| \leq\left|g_{t}\left(x, v_{\lambda}\right)\right|\left|U_{\lambda}\right| \text {. }
$$

By assumption $\widetilde{\left(g_{1}\right)}$,

$$
\left|g_{t}\left(x, v_{\lambda}\right)\right|\left|U_{\lambda}\right| \leq b(x)\left|v_{\lambda}\right|^{p}\left|U_{\lambda}\right| \leq 2^{p-1} b(x)\left(\left\|u_{\lambda}\right\|_{\infty}^{p}+\left\|\bar{u}_{\lambda}\right\|_{\infty}^{p}\right)\left|U_{\lambda}\right| .
$$

Therefore, (4.28) implies that $U$ solves $-\Delta U=\lambda_{i} U$ in $H_{0}^{1}(\Omega)$. Indeed,

$$
\begin{gathered}
\left|\int_{\Omega} \frac{g\left(x, u_{\lambda}\right)-g\left(x, \bar{u}_{\lambda}\right)}{\left\|U_{\lambda}\right\|} \phi \mathrm{d} x\right| \leq 2^{p-1}\left(\left\|u_{\lambda}\right\|_{\infty}^{p}+\left\|\bar{u}_{\lambda}\right\|_{\infty}^{p}\right) \int_{\Omega} b(x) \frac{\left|U_{\lambda}\right|}{\left\|U_{\lambda}\right\|}|\phi| \mathrm{d} x \\
\leq 2^{p-1}\left(\left\|u_{\lambda}\right\|_{\infty}^{p}+\left\|\bar{u}_{\lambda}\right\|_{\infty}^{p}\right) \frac{\left\|U_{\lambda}\right\|_{2^{*}}}{\left\|U_{\lambda}\right\|}\|b\|_{N / 2}\|\phi\|_{2^{*}},
\end{gathered}
$$

and since $u_{\lambda} \rightarrow 0$ uniformly, the assertion is proved, for $\left\|U_{\lambda}\right\|_{2^{*}} \leq \gamma\left\|U_{\lambda}\right\|$ by Sobolev inequality (we wrote the estimates in the case $N \geq 3$, the case $N<3$ being analogous). Thus there exists $t \in \mathbb{R}$ such that $U=t e_{i}$. But from (4.28) we also get

$$
\int_{\Omega} \frac{\left|D U_{\lambda}\right|^{2}}{\left\|U_{\lambda}\right\|^{2}} \mathrm{~d} x-\lambda \int_{\Omega} \frac{U_{\lambda}^{2}}{\left\|U_{\lambda}\right\|^{2}} \mathrm{~d} x=\int_{\Omega} \frac{g\left(x, u_{\lambda}\right)-g\left(x, \bar{u}_{\lambda}\right)}{\left\|U_{\lambda}\right\|^{2}} U_{\lambda} \mathrm{d} x .
$$

Reasoning as above,

$$
\left|\int_{\Omega} \frac{g\left(x, u_{\lambda}\right)-g\left(x, \bar{u}_{\lambda}\right)}{\left\|U_{\lambda}\right\|^{2}} U_{\lambda} \mathrm{d} x\right| \leq 2^{p-1}\left(\left\|u_{\lambda}\right\|_{\infty}^{p}+\left\|\bar{u}_{\lambda}\right\|_{\infty}^{p}\right) \frac{\left\|U_{\lambda}\right\|_{2^{*}}^{2}}{\left\|U_{\lambda}\right\|^{2}}\|b\|_{N / 2} .
$$

Thus, passing to the limit,

$$
1=\lambda_{i} \int_{\Omega} U^{2} \mathrm{~d} x
$$


and therefore

$$
\int_{\Omega}|D U|^{2} \mathrm{~d} x=\lambda_{i} \int_{\Omega} U^{2} \mathrm{~d} x=1,
$$

so that $U_{\lambda} /\left\|U_{\lambda}\right\| \rightarrow U$ strongly in $H_{0}^{1}(\Omega)$ and $t= \pm \frac{1}{\sqrt{\lambda_{i}}}$.

Thus there exists $e \in E_{\lambda_{i}}$ such that

$$
\frac{U_{\lambda}}{\left\|U_{\lambda}\right\|} \longrightarrow \frac{1}{\sqrt{\lambda_{i}}} e \quad \text { in } H_{0}^{1}(\Omega) \text { and a.e. in } \Omega \text {. }
$$

Since $e$ changes sign and its nodal line is not closed, and since $\Omega$ is symmetric w.r.t. every $x_{j}$-axes, there exists $x=\left(x_{1}, \ldots, x_{N}\right) \in \Omega$ and $j \in\{1, \ldots, N\}$ such that $e(x)>0$ and $e(\bar{x})<0$, where we set $\bar{x}=$ $\left(x_{i}, \ldots,-x_{j}, \ldots, x_{N}\right)$. Moreover, by $(4.29)$, we can also suppose that

$$
\frac{U_{\lambda}(x)}{\left\|U_{\lambda}\right\|} \rightarrow \frac{1}{\sqrt{\lambda_{i}}} e(x)>0
$$

and

$$
\frac{U_{\lambda}(\bar{x})}{\left\|U_{\lambda}\right\|} \rightarrow \frac{1}{\sqrt{\lambda_{i}}} e(\bar{x})<0 .
$$

If $\lambda$ is sufficiently close to $\lambda_{i}$, by (4.30) we would have

$$
u_{\lambda}(x)>\bar{u}_{\lambda}(x)=-u_{\lambda}\left(x_{1}, \ldots,-x_{j}, \ldots, x_{n}\right)
$$

and by (4.31)

$$
u_{\lambda}\left(x_{1}, \ldots,-x_{j}, \ldots, x_{n}\right)<\bar{u}_{\lambda}(x)=-u_{\lambda}(x) .
$$

Therefore, (4.32) and (4.33) give $u_{\lambda}(x)>u_{\lambda}(x)$ and a contradiction arises.

Then $U_{\lambda} \equiv 0$.

We are now able to conclude.

Proof of Theorem 2.2. By assumption $g\left(x_{1},-x_{2},-s\right)=-g(x, s)$, so that Lemma 4.1 implies that both $u_{\lambda}\left(x_{1}, x_{2}\right)$ and $-u_{\lambda}\left(x_{1},-x_{2}\right)$ solve problem (1.2). By Theorem 1.2 in [11], since $\Omega \subset \mathbb{R}^{2}$ is convex and symmetric w.r.t. $x_{1}$ and $x_{2}$, any second eigenfunction of $-\Delta$ in $H_{0}^{1}(\Omega)$ changes sign exactly once and the eigenspace associated to $\lambda_{2}$ is spanned by eigenfunctions each of which is odd in one variable and even in the other one; thus any linear combination of them has its nodal line intersecting $\partial \Omega$. Then, by Lemma 4.2 applied for $i=N=2$, if $\lambda \in\left(\lambda_{2}-\tau_{2}^{\prime}, \lambda_{2}\right)$ we get $u_{\lambda}\left(x_{1}, x_{2}\right)=-u_{\lambda}\left(x_{1},-x_{2}\right)$.

Acknowledgements. The author wishes to thank Prof. F. Pacella for suggesting the problem of symmetry for solutions near resonance.

Moreover, the author takes the opportunity to thank the anonymous referee for the careful reading and for the helpful comments, which improved the presentation of the paper.

\section{REFERENCES}

[1] A. Ambrosetti and P.H. Rabinowitz, Dual variational methods in critical point theory and applications. J. Funct. Anal. 14 (1973) 349-381.

[2] M. Balabane, J. Dolbeault and H. Ounaies, Nodal solutions for a sublinear elliptic equation. Nonlinear Analysis TMA 52 (2003) 219-237.

[3] A. Bahri and P.L. Lions, Solutions of superlinear elliptic equations and their Morse indices. Comm. Pure Appl. Math. 45 (1992) 1205-1215.

[4] T. Bartsch, K.C. Chang and Z.Q. Wang, On the Morse indices of sign changing solutions of nonlinear elliptic problems. Math. Z. 233 (2000) 655-677. 
[5] T. Bartsch, Z. Liu and T. Weth, Sign changing solutions of superlinear Schrödinger equation. Comm. Partial Differ. Equ. 29 (2004) 25-42.

[6] T. Bartsch and T. Weth, A note on additional properties of sign changing solutions to superlinear elliptic equations. Topol. Methods Nonlinear Anal. 22 (2003) 1-14.

[7] T. Bartsch and T. Weth, Three nodal solutions of singularly perturbed elliptic equations on domains without topology. Ann. Inst. H. Poincaré Anal. Non Linéaire 22 (2005) 259-281.

[8] V. Benci and D. Fortunato, A remark on the nodal regions of the solutions of some superlinear elliptic equations. Proc. Roy. Soc. Edinburgh Sect. A 111 (1989) 123-128.

[9] H. Brezis and T. Kato, Remarks on the Scrödinger operator with singular complex potentials. J. Pure Appl. Math. 33 (1980) $137-151$.

[10] A. Castro, J. Cossio and J.M. Neuberger, A minmax principle, index of the critical point, and existence of sign-changing solutions to elliptic boundary value problems. Electron. J. Differ. Equ. 2 (1998) 18.

[11] L. Damascelli, On the nodal set of the second eigenfunction of the Laplacian in symmetric domains in $\mathbb{R}^{N}$. Atti Accad. Naz. Lincei Cl. Sci. Fis. Mat. Natur. Rend. Lincei (9) Mat. Appl. 11 (2000) 175-181.

[12] L. Damascelli, M. Grossi and F. Pacella, Qualitative properties of positive solutions of semilinear elliptic equations in symmetric domains via the maximum principle. Ann. Inst. H. Poincaré. Anal. Non Linéaire 16 (1999) 631-652.

[13] L. Damascelli and F. Pacella, Monotonicity and symmetry of solutions of $p$-Laplace equations, $1<p<2$, via the moving plane method. Ann. Scuola Norm. Sup. Pisa Cl. Sci. (4) 26 (1998) 689-707.

[14] L. Damascelli and F. Pacella, Monotonicity and symmetry results for $p$-Laplace equations and applications. Adv. Differential Equations 5 (2000) 1179-1200,

[15] P. Drábek and S.B. Robinson, On the Generalization of the Courant Nodal Domain Theorem. J. Differ. Equ. 181 (2002) $58-71$.

[16] M. Grossi, F. Pacella and S.L. Yadava, Symmetry results for perturbed problems and related questions. Topol. Methods Nonlinear Anal. (to appear).

[17] S.J. Li and M. Willem, Applications of local linking to critical point theory. J. Math. Anal. Appl. 189 (1995) 6-32.

[18] J. Moser, A new proof of De Giorgi's theorem. Comm. Pure Appl. Math. 13 (1960) 457-468.

[19] D. Mugnai, Multiplicity of critical points in presence of a linking: application to a superlinear boundary value problem. Nonlinear Differ. Equ. Appl. 11 (2004) 379-391.

[20] F. Pacella, Symmetry results for solutions of semilinear elliptic equations with convex nonlinearities. J. Funct. Anal. 192 (2002) 271-282

[21] P.H. Rabinowitz, Minimax methods in critical point theory with applications to differential equations. CBMS Regional Conference Series in Mathematics 65, American Mathematical Society, Providence, RI (1986).

[22] M. Struwe, Variational Methods. Applications to nonlinear partial differential equations and Hamiltonian systems. Springer-Verlag (1990).

[23] Z.Q. Wang, On a superlinear elliptic equation. Ann. Inst. H. Poincaré Anal. Non Linéaire 8 (1991) 43-57. 